\title{
Catch me if you can: targeting the mitochondrial permeability transition pore in myocardial infarction
}

\author{
A Linkermann ${ }^{\star, 1}, \mathrm{~K}_{\text {Konstantinidis }}{ }^{2}$ and RN Kitsis ${ }^{*, 3}$
}

Cell Death and Differentiation (2016) 23, 1-2; doi:10.1038/cdd.2015.151

Myocardial infarction (MI) occurs when an atherosclerotic plaque suddenly ruptures leading to formation of a thrombus that occludes a coronary artery. The resulting ischemia kills cardiomyocytes primarily through necrosis, much of which is mediated by opening of the mitochondrial permeability transition pore (mPTP) in the inner membrane. ${ }^{1}$ Standard treatment for ST-segment elevation MI (STEMI), the most damaging type of infarct, is to promptly restore myocardial perfusion by relieving the coronary obstruction, referred to as reperfusion. Although the net effect of reperfusion is to limit damage, ${ }^{2}$ the reintroduction of oxygenated blood into an ischemic zone may induce substantial additional mPTPdependent necrosis, termed reperfusion injury. ${ }^{3} \mathrm{~A}$ recent article by Cung et al. reported that cyclosporine $\mathrm{A}$, an inhibitor of MPTP opening, failed to lessen adverse outcomes from reperfusion injury. ${ }^{4}$ This negative study is of interest because it raises important questions about mechanisms of cell death in $\mathrm{Ml}$ and the creation of effective therapies to oppose them.

Nowadays acute mortality from $\mathrm{Ml}$ is 'only' $5-6 \%{ }^{2}$ Most deaths result from a second problem known as heart failure, which develops over months to years following MI (Figure 1).

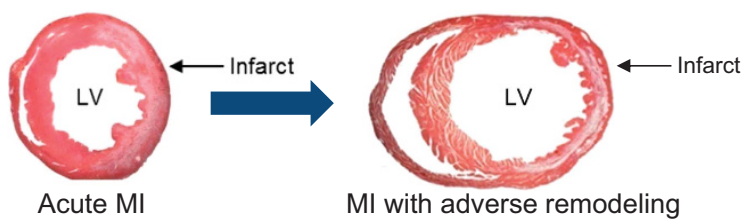

Figure 1 Post-infarct remodeling and heart failure. During Ml, cardiomyocytes in the infarct zone die primarily by necrosis, much of which is regulated. This loss of functional muscle, which is complete in $<24 \mathrm{~h}$, activates a variety of stress pathways in non-infarcted areas of the heart, which induce long-term structural and functional changes, referred to collectively as adverse remodeling. Adverse remodeling precipitates additional contractile dysfunction within the non-infarcted myocardium. The molecular and cellular basis of these events is complex and involves dysfunction of cardiomyocytes and low-level, but ongoing, attrition of these cells through apoptosis. Shown are hematoxylin and eosin-stained cross-sections of mouse hearts $3 \mathrm{~d}$ (left) and $7 \mathrm{~d}$ (right) following surgical occlusion of the left coronary artery for $1 \mathrm{~h}$ followed by reperfusion in vivo. The infarct appears white. Dilation of the left ventricular (LV) cavity at the $7 \mathrm{~d}$ time point reflects adverse remodeling. Images courtesy of Dr. Nikolaos G Frangogiannis.
Heart failure can be thought of as a weakening of the cardiac muscle that limits the effective delivery of blood to tissues throughout the body. Heart failure is one of the most common causes of death worldwide, and prior Mls are the etiology in $\sim 50 \%$ of cases.

Importantly, the size of the acute infarction is the major determinant of adverse cardiac remodeling, heart failure, and patient death. ${ }^{5}$ Timely reperfusion of STEMI using angioplasty/stenting provides significant net reductions in infarct size and improvements in clinical outcomes. ${ }^{2}$ But, the benefits of this therapy are thought to be limited by reperfusion injury. Attempts to reduce reperfusion injury have been unsuccessful - possibly because of targeting of redundant death pathways or processes that lie downstream of the actual cell death event.

Although traditionally considered passive and unregulated, recent work has established that necrosis in many contexts is actively mediated through two major pathways: ${ }^{6}$ (a) necroptosis, involving cell surface death receptors/RIPK1/RIPK3// MLKL1; and (b) mitochondrial-dependent necrosis, in which the sentinel event is $\mathrm{Ca}^{2+}$-induced opening of the MPTP. In healthy cells, MPTP is mostly in the closed state, which maintains the electrochemical gradient across the inner membrane $\left(\Delta \psi_{\mathrm{m}}\right)$ required for ATP synthesis. Increased concentrations of $\mathrm{Ca}^{2+}$ in the mitochondrial matrix, such as during ischemia, trigger opening of the pore, leading to: (a) loss of $\Delta \psi_{\mathrm{m}}$ with cessation of ATP synthesis; and (b) mitochondrial swelling and dysfunction due to influx of water into the soluterich matrix. Recent work suggests that the F0-F1 ATP synthase itself may be the channel-forming component of mPTP. ${ }^{7}$ Although not part of the channel, cyclophilin $\mathrm{D}$, a peptidyl prolyl cis-trans isomerase, sensitizes the pore to $\mathrm{Ca}^{2+}$-induced opening. Although the relevant targets of its isomerase activity are not known, mice lacking cyclophilin $D$ exhibit resistance to pore opening and demonstrate smaller infarcts following myocardial ischemia/reperfusion. ${ }^{8,9}$ Cyclosporine $\mathrm{A}$, a small molecule that binds to and inhibits cyclophilin $\mathrm{D}$, has been shown to provide similar cardioprotection in small and large animals. ${ }^{3}$

\footnotetext{
${ }^{1}$ Clinic for Nephrology and Hypertension, Christian-Albrechts-University, Kiel, Germany; ${ }^{2}$ Division of Cardiology, Department of Medicine, Johns Hopkins School of Medicine, Baltimore, MD, USA and ${ }^{3}$ Departments of Medicine (Cardiology) and Cell Biology and Wilf Family Cardiovascular Research Institute, Albert Einstein College of Medicine, Bronx, New York, NY, USA

*Corresponding author: A Linkermann, Campus Kiel Clinic for Nephrology and Hypertension, University-Hospital Schleswig-Holstein, Fleckenstr. 4, 24105 Kiel, Germany. Tel: +49 431597 7362; Fax: +49 431597 7369; E-mail: andreas.linkermann@uksh.de or RN Kitsis, Departments of Medicine (Cardiology) and Cell Biology and Wilf Family Cardiovascular Research Institute, Albert Einstein College of Medicine, 1300 Morris Park Avenue, Bronx, New York, NY 10461, USA. Tel: +1 718430 2609; Fax: +1 718430 8989; E-mail: richard.kitsis@ einstein.yu.edu
} 
In the present study, Cung et al. ${ }^{4}$ reported a multicenter, double-blind, randomized, phase III clinical trial (referred to as CIRCUS), in which cyclosporine A failed to provide benefit with respect to important clinical outcomes and adverse remodeling. To be included in the study, patients needed to present within $12 \mathrm{~h}$ of symptom onset with a STEMI involving the left anterior descending artery, and be treated with reperfusion using angioplasty/stenting. Cyclosporine $A$ (2.5 mg/kg body weight) or placebo was administered intravenously immediately before reperfusion. Among 345 patients treated with cyclosporine $A$ and 336 who received placebo, no differences were found in the primary composite outcome (death from any cause, worsening of heart failure during the initial hospitalization, re-hospitalization for heart failure, and adverse cardiac remodeling within 1 year), or in a variety of secondary outcomes that included the components of the composite.

How does one interpret this 'negative' study? In theory, the high incidence of adverse remodeling in both groups may have obscured a difference in the primary composite outcome. But, even if this were the case, the individual components of the composite also failed to show differences. Rather, the most likely reason why cyclosporine $A$ failed to ameliorate the adverse clinical and remodeling outcomes is that this drug did not appear to reduce infarct size in the current study. Infarct size was assessed using serum concentrations of creatine kinase, reflecting leakage of this protein from necrotic cardiomyocytes. Unfortunately, the cardiac-specific isoform was not measured, nor were levels of troponin I, a more sensitive marker of MI. As infarct size is the primary driver of adverse events, it is not surprising that these downstream clinical and remodeling end points were not ameliorated.

The absence of an effect of cyclosporine A on infarct size in this study contrasts with the results of an earlier phase 2 clinical trial by these investigators in which the same dose of cyclosporine A resulted in smaller infarcts as measured by creatine kinase and magnetic resonance imaging. ${ }^{10,11}$ Several caveats need to be noted, however. First, troponin I levels were measured in this earlier study, and did not differ significantly between groups. Second, the earlier study involved a relatively small number of patients. Third, and probably most importantly, reductions in infarct size by cyclosporine $A$ have not been invariably observed in humans and animals, ${ }^{12}$ an important point that will be discussed below.

A confounding factor is that the current study employed a formulation of cyclosporine A (CicloMulsion (NeuroVive Pharmaceutical $A B$, Lund, Sweden)) that differed from the one used in the previous study (Sandimmune (Novartis, Cambridge, MA, USA)). Although CidoMulsion and Sandimmune appear equivalent in terms of pharmacokinetic properties, CidoMulsion is delivered as a lipid emulsion, whereas, Sandimmune as a polyoxyethylated castor oil. Differential effects of these vehicles on mitochondria have been reported and might influence infarct size. ${ }^{4}$ The ability of CidoMulsion to reduce infarct size in animal models has not been published, although the authors noted their unpublished data that it works in mouse hearts. Whether formulation issues have a significant role in the reported results is currently unclear.

The fact that even Sandimmune has not consistently reduced infarct size, however, raises more fundamental issues. First, is cyclosporine A the best way to inhibit mPTPdependent necrosis? Regarding this question, it should be noted that this small molecule has other pharmacological actions. For example, cyclosporine $\mathrm{A}$ inhibits calcineurin, a phosphatase with multiple targets. One substrate is Drp1, which undergoes calcineurin-mediated dephosphorylation to promote mitochondrial fission. ${ }^{13}$ Accordingly, cyclosporine A may, in theory, promote a shift in mitochondrial dynamics toward the fused state. This may be significant with respect to the variability in cardioprotection provided by cyclosporine A because the fused mitochondrial state sensitizes to MPTP opening. $^{14}$

Second, although experiments using mice lacking cyclophilin $D^{8,9}$ and cyclosporine $A^{3}$ implicate mPTP opening in reperfusion injury, might other death processes provide redundancy when $\mathrm{mPTP}$ opening is inhibited? In fact, genetic and pharmacological data suggest that necroptosis may contribute to infarct size in heart and kidney following ischemia/reperfusion injury. ${ }^{15}$ Accordingly, it is possible that combined targeting of necroptosis and mPTP-dependent necrosis may result in more marked and consistent reductions in reperfusion injury. Moreover, an understanding of molecular connections between these modes of necrosis may allow the identification of a single target against which a drug could inactivate both processes.

\section{Conflict of Interest}

The authors declare no conflict of interest.

Acknowledgements. The work in the Linkermann group is funded by the German research foundation (DFG) in the Cluster of Excellence, Inflammation at interfaces (EXC 306), Kiel, Germany. The work in the Kitsis lab is supported by grants from the NIH (R01HL128071 and R01CA170911), American Heart Association, and Fondation Leducq; The Dr. Gerald and Myra Dorros Chair in Cardiovascular Disease; and the Wilf Family Cardiovascular Research Institute of the Albert Einstein College of Medicine.

1. Konstantinidis K, Whelan RS, Kitsis RN. Arterioscler Thromb Vasc Biol 2012; 32: 1552-1562.

2. American College of Emergency et al. J Am Coll Cardiol 2013; 61: e78-140.

3. Ong SB et al. J Mol Cell Cardiol 2015; 78: 23-34.

4. Cung TT et al. N Engl J Med 2015; 373: 1021-1031.

5. Miller TD et al. Circulation 1995; 92: 334-341.

6. Vanden Berghe T et al. Nat Rev Mol Cell Biol 2014; 15: 135-147.

7. Bernardi $P$ et al. Physiol Rev 2015; 95: 1111-1155.

8. Nakagawa T et al. Nature 2005; 434: 652-658

9. Baines CP et al. Nature 2005; 434: 658-662.

10. Mewton N et al. J Am Coll Cardiol 2010; 55: 1200-1205

11. Piot C et al. N Engl J Med 2008; 359: 473-481.

12. Lim WY, Messow CM, Berry C. Br J Pharmacol 2012; 165: 2034-2043.

13. Cereghetti GM et al. Proc Natl Acad Sci USA 2008; 105: 15803-15808.

14. Whelan RS et al. Proc Natl Acad Sci USA 2012; 109: 6566-6571.

15. Linkermann A et al. Proc Natl Acad Sci USA 2013; 110: 12024-12029. 